\title{
Multifractal Nature of Diesel Engine Rattle Noise in Vehicle
}

\author{
Andrzej PUCHALSKI, Iwona KOMORSKA \\ University of Technology and Humanities in Radom \\ Malczewskiego 29, 26-600 Radom, Poland; e-mail: \{andrzej.puchalski, iwona.komorska\}@uthrad.pl
}

(received March 7, 2017; accepted April 25, 2017)

\begin{abstract}
The investigation results of the emission of noises accompanying the diesel engine powertrain running in unloaded condition at low or idle speed, are presented in the hereby paper. The multifractal nature of the tested signals was confirmed by means of two groups of parameters. The proposed local parameters are based on the distribution of the probability density of measures, segregated into subsets according to pointwise Holder exponents. The obtained spectra constitute a set of multifractal measures related to singularities representing the local scaling of measures in various points of the time series. Global parameters were defined on the basis of multifractal measures of the parametrised Renyi's entropy. These are properties treating summarily the whole spectrum of the emitted noise and reflecting changes of the vibroacoustic energy levels generated by noise sources. The tests encompassing simultaneous application of both groups of parameters confirmed their efficiency in the comparative analysis as well as in the subjective assessment of the noise level generated in the drive system of vehicles with diesel engines. This opens new possibilities within the simulation range of vehicle noises, for the needs of constructing the passive and active reduction systems of the effects of the vibroacoustic energy propagation.
\end{abstract}

Keywords: mechanical noise and vibration; multifractal formalism; alpha-stable distributions.

\section{Introduction}

The complexity of the input-output relation between the noise source and the point of its recording constitutes a serious problem from the point of view of the model based vibroacoustic diagnostics (BATKO et al., 2005; 2008). More and more advanced systems of computer recording and analysis allow nonanalytical modelling, realised on the basis of signals' observations (DĄBROWSKI, 1992; DĄBROWSKI, Dziurdź, 2016; Dziurdź, 2013). The selection of the proper mathematical formalism, taking into account non-linearity, non-stationarity, as well as the arbitrary randomness of effects related to the vibroacoustic energy propagation, is the condition of the diagnostic model identification. This idea allows finding information, which cannot be obtained by means of the classic linear methods with a random forcing of the Gaussian form. Such formalism leads - in turn - to the necessity of selecting and optimising of the algorithm. These requirements are satisfied by analyses of time series based on the fractal theory. The most often utilised algorithms are based on the direct analysis of the system phase trajectory and on complexity measures such as
Lyapunov exponent, entropy, or multifractal measure (PuChalski, 2015; Puchalski, Komorska, 2014).

The characteristics of engines and machines as sources of emitting noises is based on information comprising the acoustic power and pressure level. The advanced methods of time-frequency analysis encompassing calculations of noise emissions in different spectrum bands are required for assessing the tested noise level, for performing the comparative analysis under arbitrary, real acoustic conditions, as well as for development of the noise reduction systems. The results of investigations of the vibroacoustic energy generated by the internal combustion engines were published in several papers: (BURdZIK, KonIECZNY, 2011; CHEER, Elliott, 2015; Elliott, 2010; KoniECZny et al., 2015; Komorska, Puchalski, 2013; 2015).

The noise is a primary Noise, vibration, and harshness (NVH) issue for automotive drivetrains. It is also important in the timing gears and the gears used to transmit torque to drive the accessories such as the fuel pump, oil pump, or air compressor in the diesel engine. The engine transmits non-uniform torques from the cranktrain to the powertrain and the geartrain and that causes the gear rattle noise related to the en- 
gine firing frequency (QIANFAn, 2013; SoBECKI, 2014; SZADKOWSKI, 1991).

The noise generated by the different components and mechanisms in the powertrain and geartrain play a significant role in determining the quality of the overall vehicle noise. The irregular rotation of the combustion engine cause comfort-reducing gear rattle. A gear rattle noise is caused by the consecutive tooth impacts resulting from the gearbox clearance and torque pulsation through the gear system. It is assumed that the main reasons for the increase of this kind of noise are mechanical forces generated in the gearbox mesh, when either the fuel injection is not properly adjusted or the damping of torsional vibrations by the clutch is wrong. The factors that affect the noise level include the backlash of the gears, drag torque on the driven gear, oil level, and oil viscosity. A rattle noise accompanying the engine operation at the coupled gear is weakening in the moment of gear uncoupling.

Impacts occur with greater intensity on diesel engine vehicles, because the torque irregularities are increased due to the way of the fuel ignition. These prob- lems could be solved by adopting devices such as double mass flywheels or multistage clutches and reducing gears' backlashes. Also oil used for lubrication of gears, acting as a damper, gives a positive contribution in reducing the above noises induced by impacts. Due to the trends towards down speeding and downsizing in engines, sound quality of automobiles greatly affected by gear rattle is still a problem.

The analysis of the emission of noises accompanying the diesel engine gearbox running in neutral condition with idle or low speed (i.e., idle rattle), is presented in this paper. The aim and scope of the presented research was to describe the sounds produced by these phenomena and investigate the feasibility of a multifractal analysis for gear rattle metric in transmission gears due to tooth impacts under unloaded conditions.

Noise signal of a four cylinder engine with fivespeed manual transmission of a front wheel drive automobile was examined, with focus on the gear idle rattle problem. The examples of time series from an online sound database and calculated spectra are shown, respectively, in Figs. 1 and 2.

a)

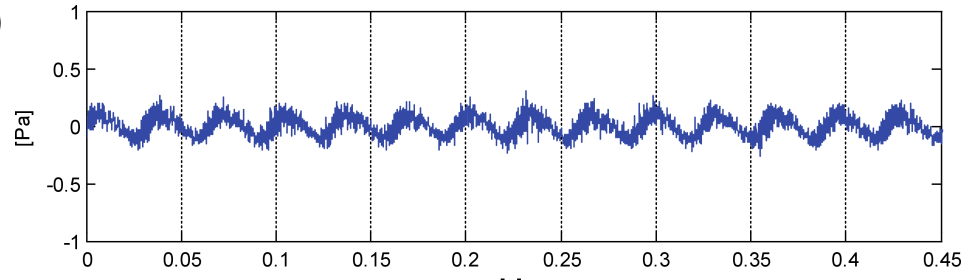

b)

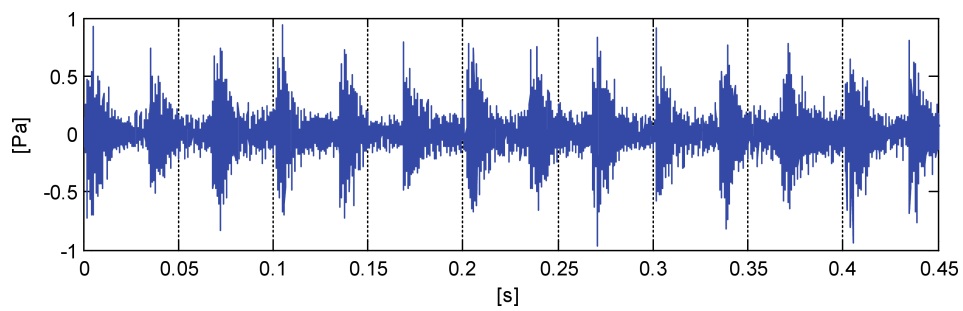

Fig. 1. Vehicle diesel engine noise: a) low rattle, b) high-excessive rattle.

a)

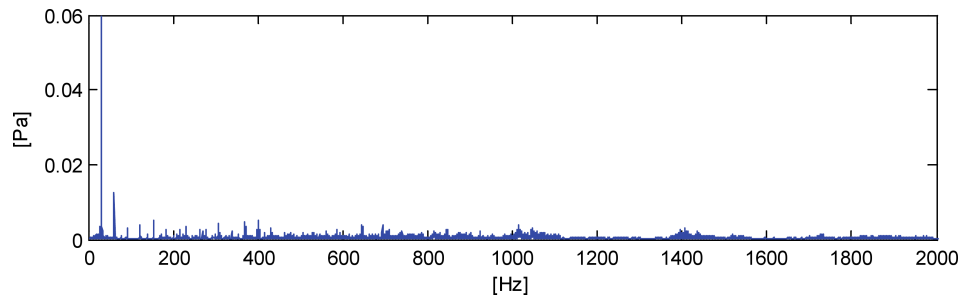

b)

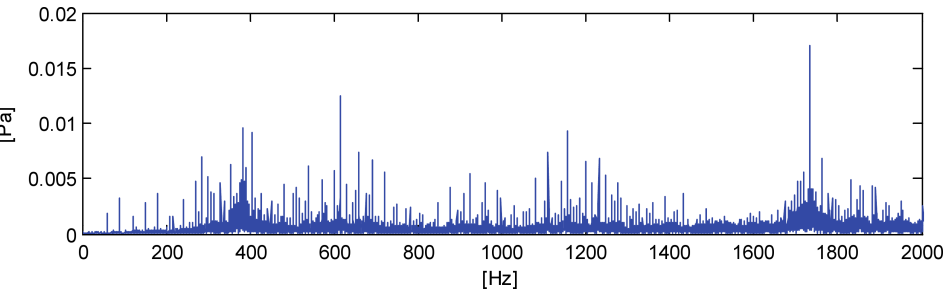

Fig. 2. Spectra of vehicle diesel engine noise: a) low rattle, b) high-excessive rattle. 
In both spectra there is a component related to the engine ignition frequency (about $30 \mathrm{~Hz}$ ) and its harmonics. It dominates in the case of "low idle rattle", whereas impulsive, broadband features can be seen in the case of "high idle rattle" which is dominated by gear-related components, modulated by output shaft speed (about $7.5 \mathrm{~Hz}$ ).

\section{Preliminaries}

Treating the tested object as a complex system of non-linear and non-stationary properties, the possibility of describing its dynamics based on experimental data in the multifractal domain was assumed. The investigation results based on box dimensions were presented. The curve dimension being the diagram of the discussed signal was assumed as the fractal dimension. The fractal dimension of the time series $D$ describes its fuzziness degree. If $L$ is a minimal number of wheels of diameter $\varepsilon$, covering the given time series, then: $L \propto \varepsilon^{-D}$. From there:

$$
D=\lim _{\varepsilon \rightarrow 0} \frac{-\lg L}{\lg (\varepsilon)} .
$$

Selfsimilar time series or time series indicating selfsimilarity properties, after integration, are described by the fractal dimensions $D$, corresponding to Hurst exponents $H$ for various scaling ranges, according to: $D=2-H$ (Butar, Kale, 2011; Hurst, 1951). The multifractal detrended fluctuations analysis MF-DFA (KANTELHARDT, 2011), based on the trend elimination from the tested $N$-samples time series, was used for the determination of Hurst exponents of the recorded data. This procedure was realised in the algorithm, leading to the determination of the power dependency of the $q$ order fluctuation:

$$
F_{q}(s) \sim s^{H(q)}
$$

where $H(q)$ is the generalised Hurst exponent.

A statistically reliable estimation of $H(q)$ coefficients requires the scale range selection within boundaries $s \in(10, N / 4)$. For monofractals exponent $H(q)=$ const. Fluctuation curves $F_{q}(s)$, determined in the experiment in two-logarithmic scale, allow to determine Hurst exponents and fractal dimensions in scale ranges of various characters of the signals' autocorrelation. The performed investigations confirmed that the fractal exponents present the properties' evolution of the complex dynamic system, as the maintenance time function. The knowledge of the singular fractal dimension is not enough for the total characteristic of the tested signal, since it is the average information related to the analysed scale range. In order to describe the dynamics of the system consisting of several intertwined fractals of various dimensions, the dimensions' spectrum, called the multifractal spectrum, should be considered. The irregularity measure, which ascribes weights to its individual parts, should be assumed for each point of the time series. The spectrum obtained due to segmentation can be treated as the multifractal dimension related to singularity representing the local measure scaling in various places of the time series. $H(q)$ exponent for multifractals is a decreasing function. For negative values of $q$ order, the generalised Hurst exponent describes scaling properties in segments of a low fluctuation level. On the contrary, if we consider positive values of $q$ order, the segments of high variances values will shape the fluctuation function. Knowledge of the generalised Hurst exponent allows to determine the generalised multiscale exponent: $\tau(q)=q H(q)-1$. The proposed local parameters of the signal are based on the probability density distribution of measures segregated into subsets according to point values of the Holder regularity exponents: $h=\frac{d}{d q} \tau(q)$. Dimensions of the singularity subsets are determined by the multifractal spectrum $f(h)$, obtained from the multiscale exponents as the Legendre's transformation.

$$
f(h)=q h-\tau(q) .
$$

Global parameters were defined on the basis of multifractal dimensions $D(q)$ of the parametrised Renyi's entropy: $I_{q}(\varepsilon)$ (ZMESKAL et al., 2013):

$$
D(q)=\lim _{\varepsilon \rightarrow 0} \frac{-I_{q}(\varepsilon)}{\ln \varepsilon}
$$

for $q \neq 1$. This exponent was approximated by using the dependence:

$$
D(q) \sim \frac{\tau(q)}{q-1} .
$$

The algorithm of determining local and global parameters of the time series on the basis of the generalised Hurst exponent is shown in Fig. 3.

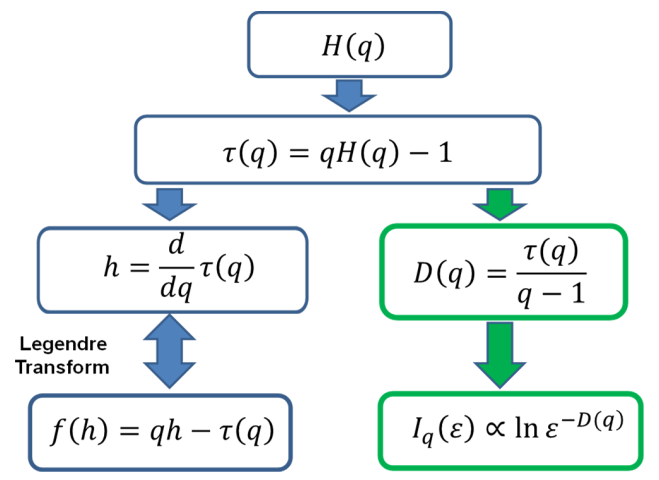

Fig. 3. Algorithm of determining local and global parameters of the noise signal.

\section{Rattle noise modelling in multifractal domain}

Confirmation of the multifractal nature of signals constitutes the multifractality level, determined by the 
singularity span of the highest and the smallest regularity exponents of the observed signal. The existence of the individual singularity indicates monofractality of the tested system. Characteristic values corresponding to various $q$ orders can be shown, when analysing multifractal dimensions of the time series entropy. For $q=1$ the informative dimension, being in accordance with the Shannon entropy definition, is obtained. When $q=2$, the fractal dimension value is called the correlation dimension of the time series. It expresses the probability of finding a pair of points of the phase attractor, reconstructed on the time series base, at the distance smaller than the determined one.

Multifractal spectra $f(h)$ of the tested noise signals, obtained due to the algorithm realisation are shown in Fig. 4, while corresponding to them multifractal exponents of entropy are shown in Fig. 5.

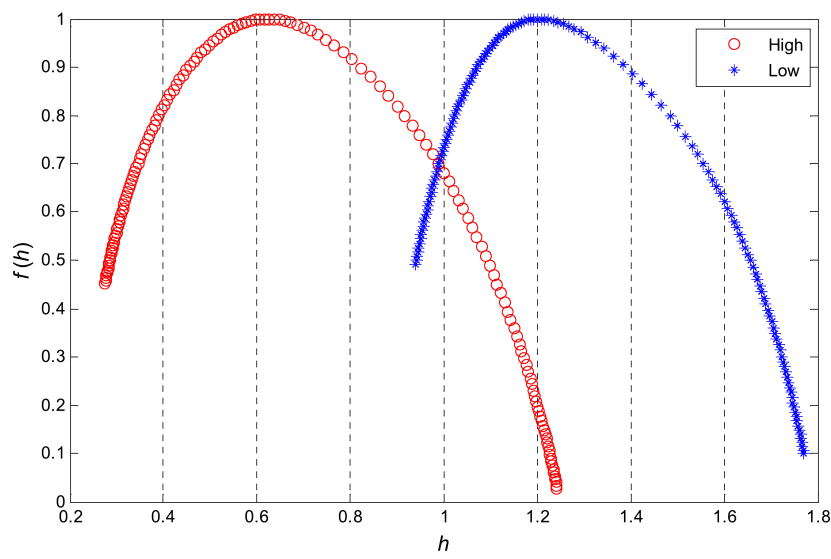

Fig. 4. Multifractal spectra of the vehicle diesel engine rattle noise.

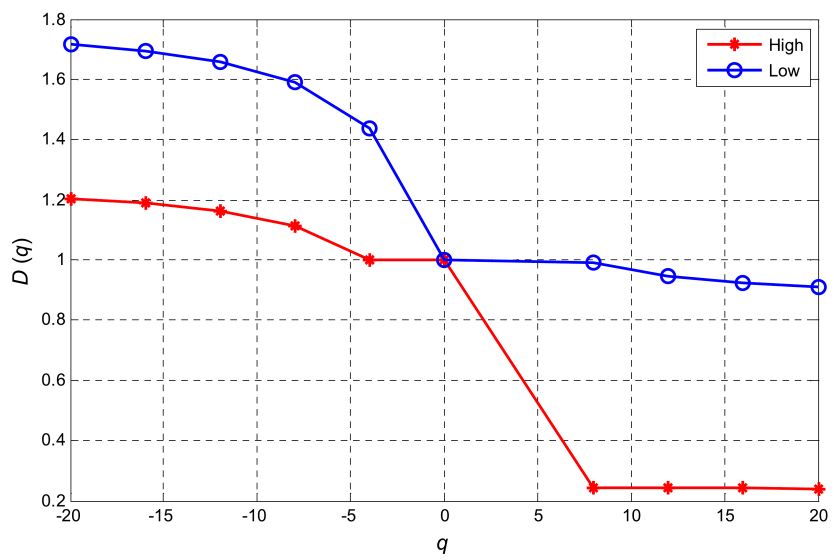

Fig. 5. Multifractal entropy dimensions of the vehicle diesel engine rattle noise.

From the point of view of the comparative analysis of the tested noise signals multifractal entropy dimensions of higher orders, $|q|>10$, have much better information value. Reny's entropy parametrised by means of the parameter $q \gg 0$ exposes the most probable events, and when $q \ll 0$ it refers to rare events, forming tails of the probability density distributions.

The probability density functions PDF of both compared noise time series are shown in Fig. 6.

a)

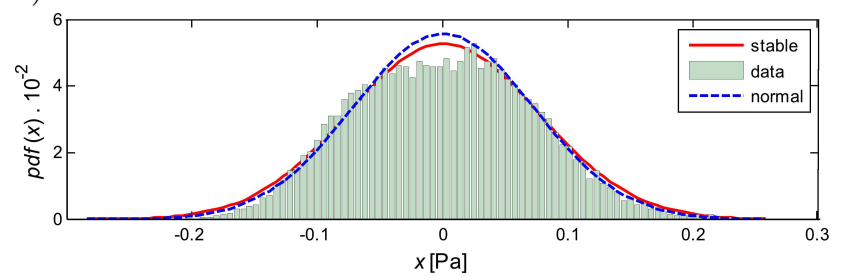

b)

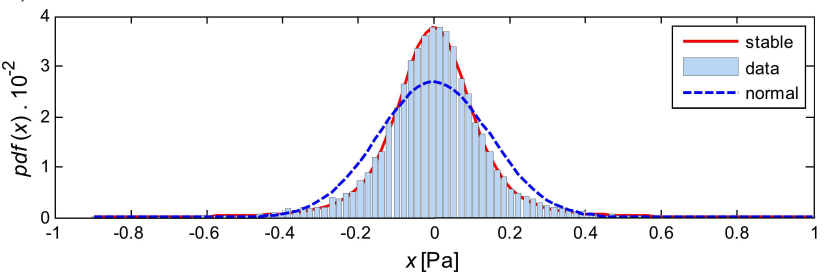

Fig. 6. PDF of tested diesel engine signals: a) low rattle noise, b) high rattle noise.

A divergence of empirical distributions from the normal one is clearly visible for the high rattle noise signal. Verification of adjusting the model of alphastable empirical distributions was performed by means of the Anderson-Darling test at the significance level 0.05 (after the preliminary graphic assessment). Modelling of heavy-tailed data enable stable distributions, which require four parameters for their complete description (BORAK et al., 2005; NOLAN, 2015; ŻAK et al., 2016; YU et al., 2013):

- stability index $\alpha \in(0,2]$,

- skewness parameter $\beta \in[-1,1]$,

- scale parameter $\gamma>0$,

- location parameter $\delta \in R$.

Index $\alpha$ determines heaviness of the distribution tail. For $\alpha=2,1$, and 0.5 we can obtain the normal, Cauchy's and Levy's distributions, respectively. Other cases of stable distributions do not have apparent forms of the density function and are defined by means of the characteristic function being the inversed Fourier transform of the density function. When $\alpha<2$ the distribution describes the asymptotic power rule. When $\beta=0$ the distribution is symmetric. Scale and location parameters, $\gamma$ and $\delta$, are equivalents of the standard deviation and average value of normal distributions.

There are several methods and algorithms of estimation of index and parameters of alpha-stable distributions on the basis of experimental data. The results obtained by means of quantile method and the maximal probability, are given in Table 1. 
Table 1. Averaged parameters of alpha-stable distributions of diesel noise signals.

\begin{tabular}{|c|c|c|}
\hline & Low rattle noise & High rattle noise \\
\hline$\alpha$ & 2.0000 & 1.5194 \\
\hline$\beta$ & 0.3439 & -0.0039 \\
\hline$\gamma$ & 0.0535 & 0.0759 \\
\hline$\delta$ & $5.8 \cdot 10^{-4}$ & $1.6 \cdot 10^{-4}$ \\
\hline
\end{tabular}

\section{Conclusion}

Analyses of multifractal spectra as well as multifractal measures of the Renyi's entropy of the tested time series of the noise emitted by the diesel engine operating in unloaded conditions confirmed the multifractal nature of the rattle noise signal. Also heavytailed distributions of the probability density are different from the normal form and indicate heavy-tailed properties, which is an essential source of multifractality. Defined multifractal parameters can be used for the estimation of the engine noise level and in the comparison analysis. Simulations of vehicle noises have been - for many years - indispensable elements of developments of passive and active systems of reducing the effects of the vibroacoustic energy propagation.

Further studies will encompass efforts of synthesising the multifractal reference model that can be used to assess gear rattle levels in a way that connects with rating the overall sound quality and human perception of diesel engines rattle as well as for the purpose of prediction of the probability of gear rattle phenomena by using the identified multifractal parameters in the task of diagnosing its constructional, technological, and maintenance reasons.

\section{References}

1. Batko W., DąBrowski Z., Engel Z., Kiciński J., Weyna S. (2005), Modern Methods of Research Vibroacoustic Processes [in Polish: Nowoczesne metody badania procesów wibroakustycznych], Institute for Sustainable Technologies, Radom, Poland.

2. Batko W., DĄBrowski Z., Kiciński J. (2008), Nonlinear Effects In Technical Diagnostics, Institute for Sustainable Technologies, Radom, Poland.

3. Borak Sz., Härdle W., Weron R. (2005), Stable Distributions, retrieved April 29, 2016, from HumboldtUniversität zu Berlin: http://prac.im.pwr.edu.pl/ 〜hugo/publ/SFB2005-008_Borak_Haerdle_Weron.pdf.

4. BurdziK R., KONIECZnY Ł. (2011), Research into noise emission by a car combustion engine exhaust system, Zeszyty naukowe AMW, Rok LII, 184, 1.

5. Butar F.B., Kale M. (2011), Fractal analysis of time series and distribution properties of Hurst exponent, Journal of Mathematical Sciences \& Mathematics Education, 5, 1, 8-19.
6. Cheer J., Elliott S.J. (2015), Multichannel control systems for the attenuation of interior road noise in vehicles, Mechanical Systems and Signal Processing, 60, 753-769.

7. DAzBrowski Z. (1992), The evaluation of the vibroacoustic activity for the needs of constructing and use of machines, Machine Dynamics Problems, 4.

8. Dąbrowski Z., Dziurdź J. (2016a), Simultaneous analysis of noise and vibration of machine in vibroacoustic diagnostics, Archives of Acoustics, 41, 4, 783789 .

9. DĄBrowski Z., Dziurdź J. (2016b), Simultaneous analysis of vibrations and noise in the task of minimizing vibroacoustic activity of machines, Archives of Acoustics, 41, 2, 303-308.

10. Dziurdź J. (2013), Analysis of nonlinear phenomena in diagnosing of the vehicle drive systems [in Polish: Analiza zjawisk nieliniowych $w$ diagnozowaniu układów napedowych pojazdów], Institute for Sustainable Technologies, Radom, Poland.

11. Elliott S.J. (2010), Active noise and vibration control in vehicles, Vehicle Noise and Vibration Refinement, Woodhead Publishing Limited, pp. 235-251.

12. Hurst H.E. (1951), Long term storage capacity of reservoirs, Transactions of the American Society of Civil Engineers, 116, 1, 770-799.

13. Kantelhardt I.W. (2011), Fractal and multifractal time series, Mathematics of Complexity and Dynamical Systems, Springer-Verlag, New York, pp. 463-487.

14. Komorska I., Puchalski A. (2013), On-board diagnostics of mechanical defects of the vehicle drive system based on the vibration signal reference model, Journal of Vibroegineering, 15, 1, 450-458.

15. Komorska I., Puchalski A. (2015), On-line diagnosis of mechanical defects of the combustion engine with principal components analysis, Journal of Vibroegineering, 17, 8, 4279-4288.

16. Konieczny L., Burdzik R., Warczek J., Czech P., Wojnar G., Mlynczak J. (2015), Determination of the effect of tire stiffness on wheel accelerations by the forced vibration test method, Journal of Vibroengineering, 17, 8, 4469-4477.

17. Nolan J.P. (2015), Stable Distributions Models for Heavy Tailed Data, retrieved June 1, 2016, from: Math/Stat Dep American Univ., http://fs2.american.edu/jpnolan/www/stable/chap1.pdf.

18. Puchalski A. (2015a), A technique for the vibration signal analysis in vehicle diagnostics, Mechanical Systems and Signal Processing, 56-57, 173-180.

19. Puchalski A. (2015b), Multiscale analysis of vibration signals in engine valve system, Journal of Vibroegineering, 17, 7, 3586-3593.

20. Puchalski A., Komorska I. (2014), Looking for vibrational measure of vehicle powertrain using multifractal analysis, Vibroengineering Procedia, 3, 351356. 
21. QIANFAN X. (2013), Noise, vibration, and harshness (NVH) in diesel engine system design, Diesel Engine System Design, Woodhead Publishing Limited, pp. 759-821.

22. Sobecki B., Davies P., Bolton J.S. (2014), Simulation of gear rattle to aid in the development of sound quality metrics for diesel engine component specification, Proceedings of 43rd International Congress on Noise Control Engineering, Melbourne, Australia.

23. SzadKowski A. (1991), Mathematical Model and Computer Simulation of Idle Gear Rattle, SAE Technical Paper 910641, doi:10.4271/910641.
24. Yu G., Li Ch., Zhang J. (2013), A new statistical modeling and detection method for rolling element bearing faults based on alpha-stable distribution, Mechanical Systems and Signal Processing, 41, 155-175.

25. Żak G., WyŁomańska A., Zimroz R. (2016), Datadriven vibration signal filtering procedure based on the $\alpha$-stable distribution, Journal of Vibroengineering, 18, 2, 826-837.

26. Zmeskal O., Dzik P., Vesely M. (2013), Entropy of fractal systems, Computers and Mathematics with Applications, 66, 135-146. 